\title{
Ultrasound for neuraxial blockade
}

\section{Karthikeyan Kallidaikurichi Srinivasan, Peter John Lee, Gabriella Iohom}

\author{
Department of Anaesthesia and Intensive Care Medicine, Cork University Hospital, Wilton, Cork, Ireland
}

\begin{abstract}
Neuraxial blockade is still largely performed as a blind procedure. Despite of developments in the type of needles used and drugs administered, the process of locating the epidural or intra-thecal space is still limited to identification of landmarks by palpation and reliance on tactile feedback of the operator. Ultrasound has provided the long needed "eye" to the procedure and has already shown promise of improving the safety and efficacy or neuraxial blocks. This review focuses on understanding the sonoanatomy of the neuraxial space, performing a systematic pre-procedural ultrasound scan, and reviewing the available evidence.
\end{abstract}

Keywords: neuraxial blockade, spinal, epidural, ultrasound, pre-procedure scanning

\section{Introduction}

There is a recent increase in the use of ultrasound (US) to facilitate spinal and epidural anesthesia. A combination of factors conspire to produce a poor US image of the neuraxis: the bony enclosure of the vertebral arches, target structures lying deep to the skin and ultrasound technology poorly suited to the task. These issues hindered the widespread use of US imaging of the neuraxis until the last decade. Rapid advancements in US imaging have addressed some of these limitations. In addition, the use of US for peripheral anaesthesia techniques has created a generation of US -proficient anaesthetists. Millions of neuraxial blocks are performed across the world, traditionally as a blind, tactile procedure. Permanent neurological injury occurs when spinal anaesthesia is administered at or above the L2-3 inter-spinous space [1]. Multiple attempts at achieving a successful neuraxial

Received 20.09.2014 Accepted 2.10.2014

Med Ultrason

2014, Vol. 16, No 4, 356-363

Corresponding author: Karthikeyan Kallidaikurichi Srinivasan Department of Anaesthesia and Intensive Care Medicine,

Cork University Hospital and University College Cork

Wilton Road, Cork, Ireland.

Phone: +353214922135

Fax: +353214546434

Email: ksrinivasan@outlook.com block are associated with an increased incidence of post dural puncture headache, paraesthesia and spinal hematoma [2-6].

Neuraxial US could potentially address these limitations thereby improving the safety of these procedures. Rapid progress has been made to the extent that routine use of pre-procedural US examination has been recommended to avoid the risk of dural puncture performed at or above the L2-3 inter-spinous space in pregnant patients [7].

Both pre-procedure and real-time US guidance have been used. This review focuses on the current use of preprocedure US guidance for neuraxial block.

\section{History}

The earliest documented use of neuraxial US dates back to 1971 by Bogin et al [8]. The authors used US to examine the anatomy of the vertebral column to aid lumbar puncture. Grau et al subsequently published a series of articles, mostly in obstetric populations, examining the role of neuraxial US, which paved the way for current research [9-15].

Real-time visualisation for para-median insertion of combined spinal-epidural needle was described by Karmakar et al in 2009 [16]. In this case series, real-time visualisation of needle was successfully used in $14 / 15$ patients studied. Since then multiple case studies and case 
series have been published on real time needle guidance in performing neuraxial blocks $[17,18]$. Recently the use of GPS guidance for spinal needle placement was studied by Brinkman et al in human cadavers and attracted much research in this area [19-27].

\section{Anatomy of neuraxial structures relevant to ultrasound}

Each vertebra consists of a vertebral body and arch. The arch is composed of pedicles, superior and inferior articular process, transverse processes and spinous process. In the lumbar region, the spinous processes are broader and less steep than in the thoracic region. Lumbar laminae are also less tilted with no overlap. In contrast, thoracic laminae are broader and they overlap. Fig 1a shows the broad and short spinous process of lumbar vertebrae compared to the long and narrow spinous process of thoracic vertebrae. In fig $1 \mathrm{~b}$ the difference in inter-laminar spaces is obvious. The thoracic inter-spinous space has steep angulation in addition to being narrow compared to the lumbar inter-spinous space. Thus the size of the window for US beam penetration is lesser in the thoracic region compared to the lumbar region. The needle trajectory for neuraxial blockade passes through the inter-spinous and inter-laminar spaces. A transverse cross section image at the level of the spinous process will not include articular or transverse processes. A transverse cross section at the level of inter-spinous space will include facet joints and transverse process but will not show laminae (fig 2).

The ligamentum flavum extends from the posterior surface of the lamina below to the anterior surface of the lamina above. It consists of elastic cartilage. The dura mater is closely related to the ligamentum flavum with the epidural space separating the two. The epidural space is a potential space and both the dura mater and ligamentum flavum are frequently seen as a single hyper-echoic structure, often called the posterior complex (PC).

The posterior surfaces of the vertebral bodies are connected by the posterior longitudinal ligament. Under US the two structures appear as the anterior complex (AC). The posterior longitudinal ligament is narrow at the level of vertebral bodies and broader at the level of intervertebral discs. The fibres of this ligament are denser and stronger compared to the anterior longitudinal ligament. It should be noted that the PC by definition should lie anterior to the transverse processes.

Various anatomical factors can impede the visualisation of neuraxial structures: a narrow inter-spinous space, thickening and calcification of inter-spinous ligaments, facet joint hypertrophy, and the absence of fusion of ligamentum flavum in the midline.

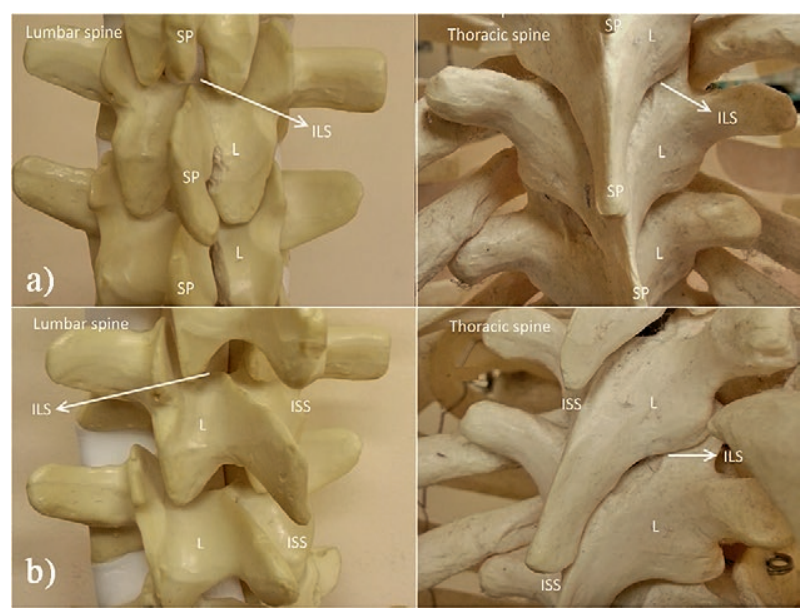

Fig 1. Lumbar and thoracic vertebrae, anatomical aspect: a) posterior view; b) lateral view. SP - spinous process, L - lamina, ILS - interlaminar space, ISS - interspinous space.

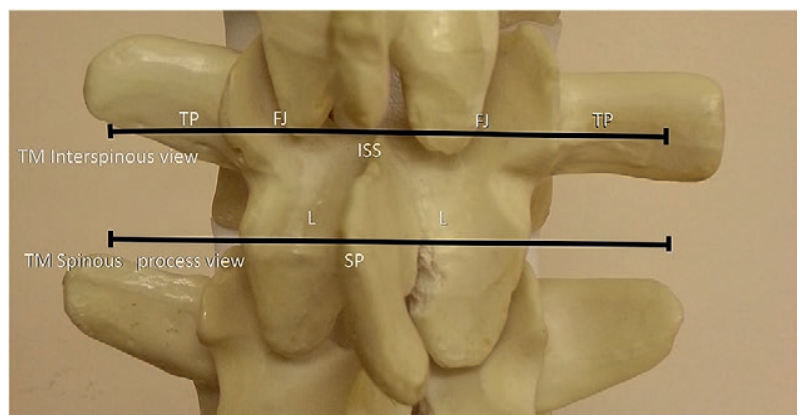

Fig 2. Structures encountered at two different cross sectional levels: ISS - inter-spinous space, L - lamina, SP - spinous process, $\mathrm{FJ}$ - facet joint, $\mathrm{TP}$ - transverse process

\section{Clinical sonoanatomy}

\section{Equipment}

A low frequency (2 to $5 \mathrm{MHz}$ ) curvilinear probe is preferred to visualise the neuraxial structures due to the depth at which they are located. Depth $(8 \mathrm{~cm}$ to start with), focus point and gain are adjusted as appropriate. Because of the limitations of the bony window, it is essential that ideal conditions for scanning are maintained including plenty of US gel, dimming the room lights and positioning of the operator. Higher frequency linear and hockey stick (7-13 MHz) probes may be used in the paediatric population because of the lesser distance from skin to neuraxis and limited ossification, especially in infants less than 6 months.

\section{Sonoanatomy}

Neuraxial US scanning is done in two planes - sagittal and transverse. Due to the broader bony window, lumbar neuraxial structures are visualised better compared to the 
thoracic region. Bony structures (spinous process, laminae, transverse processes and vertebral body) are hyper-echoic and are seen as bright white structures. Ligamentous structures (inter-spinous ligament, posterior longitudinal ligament and ligamentum flavum) and membranes (dura mater) are less hyper-echoic compared to bone. Fat, muscle and fluid are progressively hypo-echoic and seen as darker structures. Pattern recognition plays a very important role in identification of structures. The following description refers to pre-procedure neuraxial ultrasound examination of the lumbar region unless specified otherwise.

\section{Sagittal views}

In this view the long axis of the probe is placed in the sagittal plane lateral to midline (fig 3a). Different views may be obtained, depending on the position of the probe (fig 3b).

When the probe is placed approximately $3 \mathrm{~cm}$ lateral to midline the transverse processes are seen as hyperechoic lines (fig 4a). These are short white lines with a slight curve. Superficial to the transverse process one can see the erector spinae muscles. Psoas muscles and peritoneum can be seen deeper to the transverse process. It is important to note the depth at which the transverse processes are seen. As one moves the probe medially, the structures encountered such as facet joints and laminae will be more superficial.

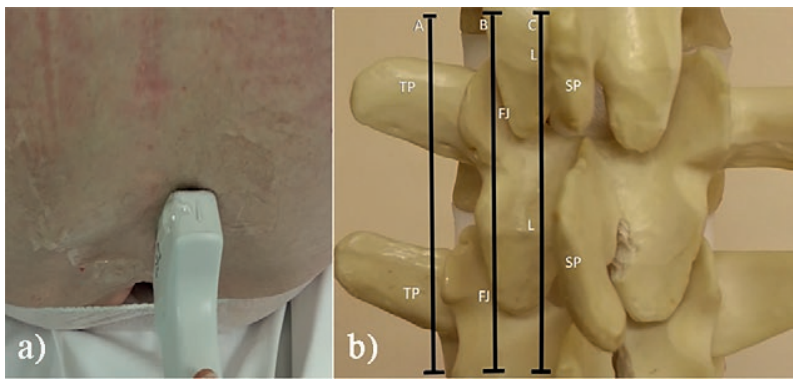

Fig 3. Para-sagittal (PS) a) probe position on a patient; b) anatomical planes of interest. SP - spinous process, $\mathrm{L}$ - lamina, FJ - facet joint, TP - transverse process; line A - PS transverse process view, line B - PS facet joint view, line C - PS laminar view.
As the probe is moved medially the facet joints are brought into view (fig $4 \mathrm{~b}$ ). It is important to note that all these structures are visualised within a few centimetres and hence movement of the probe medially should be slow and deliberate to carefully observe pattern changes. The facet joints are seen as near-continuous wavy white structures with humps (described as the "camel hump sign"). The gap between the lines is the facet joint between the superior and inferior articular processes. On further slight medial movement of the probe, the laminae are seen as slanting white lines with the alignment resembling saw teeth or horse heads arranged one after the other (fig 4c). With a slight medial tilt of the probe (towards the median sagittal plane), the inter-laminar window allows visualisation of neuraxial structures. Medial angulation in this position, described as the parasagittal oblique view (PSO), is important as it gives the best chance to visualise the neuraxial structures (fig 5a). Structures thus visualized include lamina, posterior complex (ligamentum flavum and dura mater), hypo-echoic spinal canal and the anterior complex (posterior longitudinal ligament and posterior surface of the vertebral body). In some individuals the epidural space may be seen as a small gap between the ligamentum flavum and the dura mater within the PC. This PSO view consistently provides better visualisation of neuraxial structures compared to transverse median view due to a better bony window (fig $5 \mathrm{~b}, \mathrm{c}$ ).

In addition to visualising the structures, the PSO view is also used to identify the level of the inter-laminar space. The sacrum is identified by placing the probe at the level of posterior superior iliac spine in PSO view orientation. The sacrum is identified as a continuous convex hyperechoic line (fig 5d). Due to the dorsal tilt of the sacrum, the caudal portion is superficial and the cranial portion is deeper. If a convex hyper-echoic line is seen with cranial portion superficial and caudal portion deeper, it means that either the probe orientation is incorrect or that the probe position is too caudal in the vertebral column. The lumbo-sacral junction may then be identified as the first

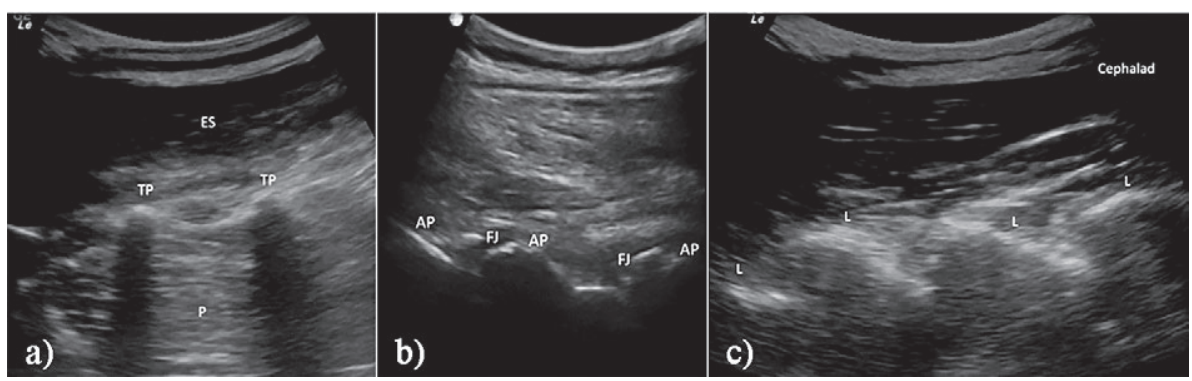

Fig 4. Para-sagittal longitudinal view of a) transverse processes; b) facet joints; c) laminae. $\mathrm{ES}$ - erector spinae muscle, $\mathrm{TP}$ - transverse process, $\mathrm{P}$ - psoas muscle, $\mathrm{FJ}$ - facet joint, $\mathrm{AP}$ - 


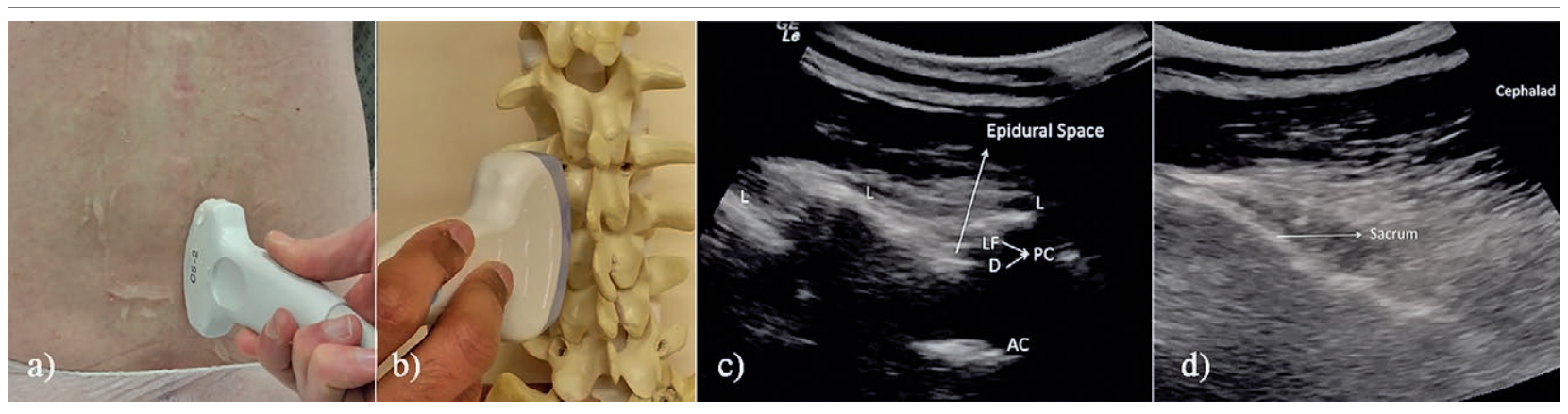

Fig 5. Para-sagittal oblique (PSO) a) probe position on a patient; b) probe position on a spine model; c) ultrasound view of lumbar spine; d) ultrasound view of sacrum. L - lamina, LF - ligamentum flavum, D - dura mater, PC - posterior complex, AC - anterior complex, S- sacrum.

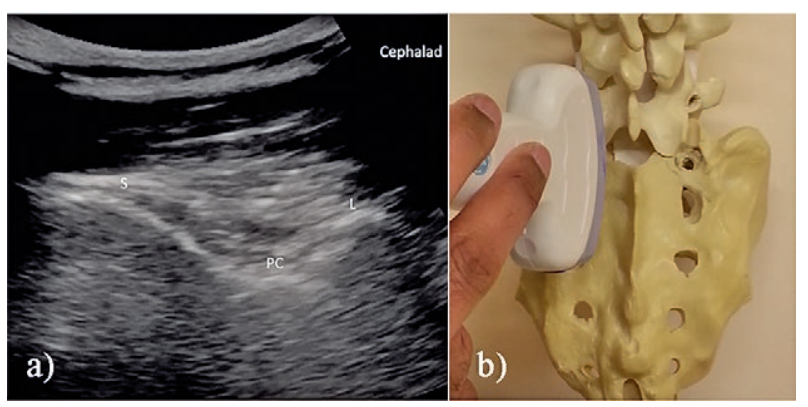

Fig 6. Para-sagittal oblique (PSO) a) ultrasound view of L5S1; b) probe position on a spine model. $\mathrm{S}$ - sacrum, L - lamina, $\mathrm{PC}$ - posterior complex

inter-laminar space between the sacrum and L5 lamina by sliding the probe cephalad (fig $6 a, b$ ). It is important to make sure that the structures visualised cephalad to the sacrum are the laminae of the lumbar vertebral body and not facets or transverse process. The key is slow and deliberate medio-lateral movement of the probe and pattern recognition. Once the L5-S1 inter-laminar space is identified, further spaces are identified by sliding the probe cephalad. Alternatively, the vertebral level may be determined by identifying the twelfth thoracic vertebra based on its articulation with the last rib, and thereafter moving the probe in a caudal direction. Note that while the probe is in the para-median sagittal position, the hyper-echoic structures seen are the laminae. The inexperienced practitioner may confuse these with the spinous processes.

From the PSO view, if one eliminates the medial tilt and moves the probe medially, it places the probe directly above the spinous process. In this view the visibility of neuraxial structures are almost impossible due to the dense hyper-echoic shadows underlying the spinous process. This view has minimal utility in identifying neuraxial structures but can be used to mark the tip of the spinous process which may be useful in patients with scoliosis.

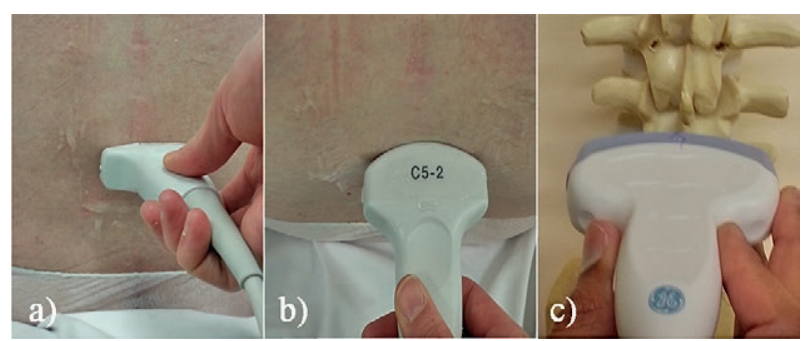

Fig 7. Transverse median (TM) a) probe position on a patient; b) cephalad angulation of the probe; c) probe position on a spine model.

\section{Transverse median views}

To obtain transverse views, the long border of the probe is placed in the transverse plane (fig 7a) and angulated cephalad to replicate the direction of the spinous processes (fig $7 \mathrm{~b}, \mathrm{c}$ ). In TM view, it is important for the operator to memorise the cephalad tilt of the probe needed to get the best view of PC and AC. This will aid the direction of advancement of the spinal or epidural needle. Depending on the position of the probe two images may be obtained.

On placing the probe directly above the spinous process (fig 8a), they are seen as hyper-echoic structures with laminae on either side (fig 8b). Due to high echogenicity of bone, structures beneath the spinous process are not seen. On moving the probe slightly cephalad or caudad, one can see the inter-spinous ligaments (fig 9a). As the echogenicity of these structures are less than spinous process it allows us to visualise other neuraxial structures. Due to the way the spinous processes and the vertebrae are arranged, scanning at this level does not show the laminae but instead the facet joints and transverse processes are seen on either side of the midline as hyper-echoic structures (fig 9b). Once this view is obtained, due to the angulation of the spinous processes, 


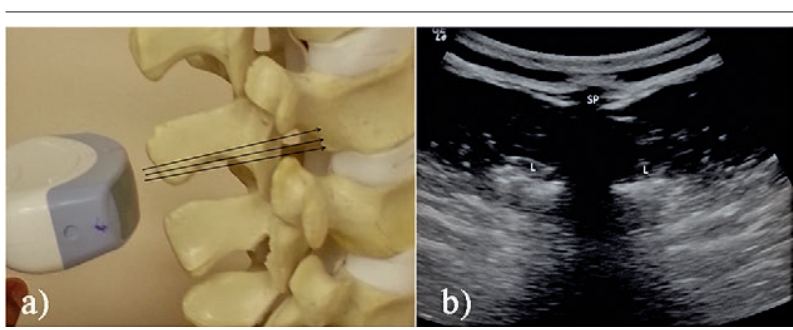

Fig 8. Transverse median cross section at the level of a lumbar spinous process a) beam path on a spine model; b) ultrasound view. $\mathrm{SP}$ - spinous process, $\mathrm{L}$ - lamina.

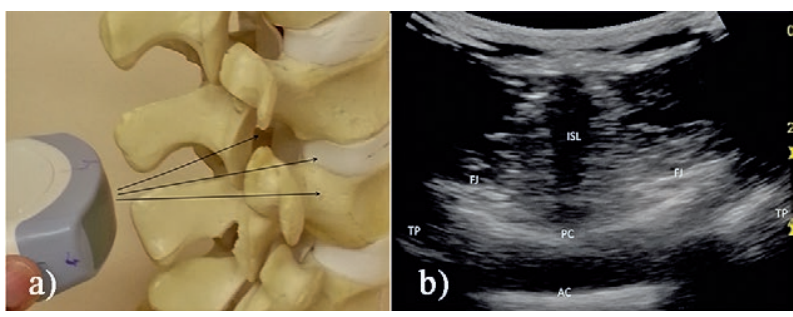

Fig 9. Transverse median cross section at the level of a lumbar inter-spinous space a) beam path on a spine model; b) ultrasound view. ISL - inter-spinous ligament, FJ - facet joint, TP - transverse process, $\mathrm{PC}$ - posterior complex, $\mathrm{AC}$ - anterior complex.

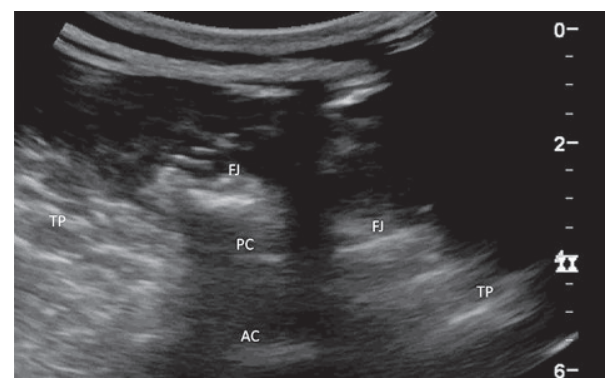

Fig 10. Transverse median view in a scoliotic spine. FJ - facet joint, $\mathrm{PC}$ - posterior complex, TP - transverse process, AC anterior chamber. Note that neither the facet joints nor the transverse processes are symmetrical. In these cases one should adjust the medio-lateral probe angulation till they are symmetrical to obtain the orientation of the vertebrae.

a slight cephalad tilt of the probe might be necessary to bring the $\mathrm{PC}$ and $\mathrm{AC}$ into view. These are seen typically as two hyper-echoic lines with the intervening spinal canal seen sometimes as a hypo-echoic structure. Their visibility may not be as good as in PSO view due to the limitations of the bony window and scattering of the ultrasound beam by the irregular surface of the bone, especially in the presence of facet joint hypertrophy. In some cases one can either visualise the $\mathrm{PC}$ or AC. The fact that the $\mathrm{AC}$ lies anterior to the transverse processes should help in identifying the structures seen.

In scoliotic individuals the neuraxial structures may not be aligned symmetrically (fig 10). Hence, in addition to the cephalad tilt, the probe has to be tilted medio-laterally to identify the direction in which vertebrae are oriented. This is done by rotating the probe medio-laterally until the facet joints and transverse processes are placed symmetrically on the screen. In such cases both the cephalad and medio-lateral angulation of the probe should be recalled when advancing the needle.

\section{Positioning}

The patient can be positioned in either sitting or lateral position. It is preferable to scan and mark the back in the exact position that neuraxial blockade will be administered i.e. arched back position. Due to the depth of the epidural and intra-thecal space, any deviation in the skin markings, even by few millimetres can lead to failure in locating the space. Inaccuracy of skin markings has been shown to be the most common mistake made by novices while learning neuraxial ultrasound [28].

\section{Skin markings for needle entry site}

This is done in a systematic manner to improve the accuracy.

Step 1: Identification of sacrum and inter-laminar spaces in PSO view

The ultrasound probe is placed in a sagittal plane medial to the posterior superior iliac spine. Once the sacrum is identified, the probe is moved cephalad to identify other inter-laminar spaces. In each inter-laminar space, the best possible view of anterior and posterior complex is obtained. With the inter-laminar space in the middle of the screen, the middle of the medial side of the long border of the probe is marked (fig 11a). Two to three spaces are thus marked.

Step 2: Selecting the best inter-spinous space on TM view

At each selected inter-laminar space, the probe is turned 90 degrees to obtain a transverse median view of AC and PC. Once the appropriate inter-laminar space is in the middle of the ultrasound screen, the midpoint of the long border of the probe and the midpoint of the short border of the probe is marked. As the ultrasound beam emanates from the middle of the probe, the intersection of the two markings should correspond to the needle entry point (fig 11b). Good visualisation of both $\mathrm{AC}$ and $\mathrm{PC}$ in transverse median view corresponds to successful administration of spinal anaesthetic in that level. However this has not been the case with PSO views [29]. Hence the inter-spinous space at which the best views of $\mathrm{AC}$ and PC is obtained in TM view should be selected. 


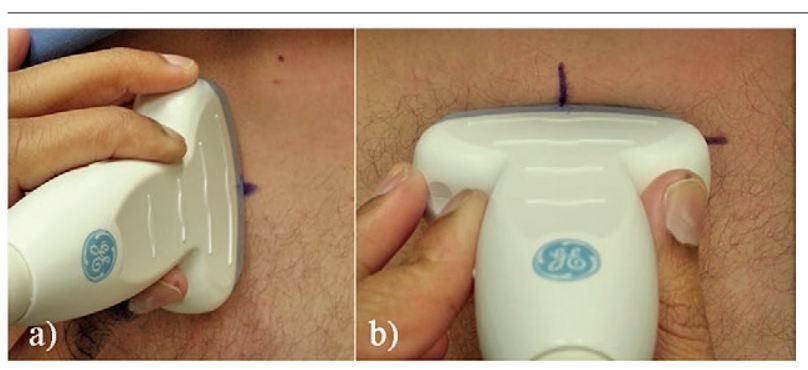

Fig 11. Skin markings prior to the neuraxial anaesthetic technique once the best image of the target has been obtained and centred in a) para-sagittal oblique view; b) transverse median view

Step 3: Angle and depth of insertion of spinal needle During skin markings for transverse median view, the cephalad angulation of the probe needed to obtain the best view of ligamentum flavum and dura mater is noted. Also the medio-lateral angle is adjusted till the facet joint and transverse processes are symmetrical on the screen (as described earlier). Both angles will have to be remembered by the operator.

The depth of the ligamentum flavum-dura mater complex (PC) from skin on ultrasound measurement has been shown to be accurate in multiple studies [18,30-32].

\section{Advantages of neuraxial ultrasound}

Pre-procedure neuraxial US scanning done by experts has been shown to increase success rates in trainee anaesthetist [33] . In patients with difficult surface landmarks (BMI $>35$, poorly palpable surface landmarks, moderate to severe lumbar scoliosis and previous lumbar spinal surgery) US has been shown to improve the success rates of spinal anaesthesia [30].

Landmark based estimation of inter-spinous space, even in experienced practitioners, has been shown to be accurate in only $39 \%$ of the time [34]. Ultrasound helps to improve accuracy. Compared with other standard modalities (MRI [35], CT [25] or plain X-rays [36]), US has been shown to be accurate up to $76 \%$ of the time. With modern US machines and adequate training, accuracy rates of up to $90 \%$ have been described [25]. Certain congenital anomalies such as sacralisation of lumbar vertebrae and lumbarisation of sacral vertebrae (which occur in up to $12 \%$ of general population [37]) can only be reliably identified by plain films.

A difference of 5 to $15 \mathrm{~mm}$ in the depth has been observed between US estimation and actual depth $[18,32,38]$. This can be attributed to tissue compression by the US probe, or an US beam angle different to that used for insertion of the spinal or epidural needle. Estimation of depth can allow one to choose appropriate needle length prior to the procedure. It may also help to avoid dural tap during epidural insertion.

Pre-procedure scanning has also shown to have an $85 \%$ positive predictive value for successful dural puncture [29]. If one can see both $\mathrm{AC}$ and PC in TM view, it indicates that there is a clear path for the US beam to pass, and hence the likelihood of inserting a spinal or epidural needle into the intended space is higher. Multiple scoring systems have been developed to assess the degree of difficulty in any given inter-spinous space [39] but the bottom line is to select the inter-spinous space with the best possible TM view of AC and PC.

\section{Limitations}

The technique of neuraxial US is a relatively new development. Due to the limited bony window, the learning curve is steep [28]. Another limitation of neuraxial US is that images are difficult to obtain in patients with difficult anatomy, in whom one might expect US to offer most benefit. Although imaging can be challenging in this group of patients, it has been shown that in experienced hands, neuraxial US is beneficial [30].

The neurotoxicity of US gel is unclear [40,41]. The gel must be cleaned away from the site of needle insertion and strict asepsis maintained. This can be difficult if real time imaging is used.

If meticulous care is not taken during the skin markings, it can lead to unsuccessful block. Factors such as a change in patient position between skin marking and needle placement, mis-estimation of the middle of the probe, movement of skin and subcutaneous tissues during probe placement (especially in elderly and obese patients), the necessity to remember cephalad and medio-lateral angles of the probe can all lead to minor inaccuracies. Due to the depth of the neuraxial structures, any minor change in skin markings or changes in angle of insertion can lead to exaggerated changes to the final position of the needle tip.

\section{Future trends}

The use of real time US-guided spinal anaesthesia has been advocated to improve the success rates. To date, this has been only demonstrated in case series and case reports $[17,21,42]$. Its use may be limited by the requirement for wide bore needles and the technical difficulties associated with simultaneous US scanning and needle advancement [23]. Studies comparing real-time with preprocedure scanning are needed to confirm the utility of this technique.

Real time volumetric three dimensional imaging of the spine using a hand held device has been shown to 
be a feasible technique in a recent study [43]. The utility of this in performing neuraxial blocks has yet to be ascertained.

\section{Conclusion}

The use of US to guide performance of neuraxial blocks is a relatively new development, yet much research is currently underway on the topic. Advances in technology have greatly helped to turn the shadows of the bony vertebral column into internal landmarks. Although some limitations remain, there is promise of improvements in the safety and efficacy of neuraxial blocks using US. Technically, the importance of pattern recognition and meticulous skin markings cannot be overstated. Neuraxial scanning, even if not performed routinely on all patients, may soon be considered the standard of care in certain patient populations.

\section{Conflict of interest: none}

\section{References}

1. Reynolds F. Damage to the conus medullaris following spinal anaesthesia. Anaesthesia 2001; 56: 238-247.

2. de Filho GR, Gomes HP, da Fonseca MH, Hoffman JC, Pederneiras SG, Garcia JH. Predictors of successful neuraxial block: a prospective study. Eur J Anaesthesiol 2002; 19: 447-451.

3. Auroy Y, Narchi P, Messiah A, Litt L, Rouvier B, Samii K. Serious complications related to regional anesthesia: results of a prospective survey in France. Anesthesiology 1997; 87: 479-486.

4. Harrison DA, Langham BT. Spinal anaesthesia for urological surgery. A survey of failure rate, postdural puncture headache and patient satisfaction. Anaesthesia 1992; 47: 902-903.

5. de Seze MP, Sztark F, Janvier G, Joseph PA. Severe and long-lasting complications of the nerve root and spinal cord after central neuraxial blockade. Anesth Analg 2007; 104: 975-979.

6. Vandermeulen EP, Van Aken H, Vermylen J. Anticoagulants and spinal-epidural anesthesia. Anesth Analg 1994; 79: 1165-1177.

7. Lee AJ, Ranasinghe JS, Chehade JM, et al. Ultrasound assessment of the vertebral level of the intercristal line in pregnancy. Anesth Analg 2011; 113: 559-564.

8. Bogin IN, Stulin ID. Application of the method of 2-dimensional echospondylography for determining landmarks in lumbar punctures. Zh Nevropatol Psikhiatr Im S S Korsakova 1971; 71: 1810-1811.

9. Grau T, Leipold RW, Delorme S, Martin E, Motsch J. Ultrasound imaging of the thoracic epidural space. Reg Anesth Pain Med 2002; 27: 200-206.
10. Grau T, Leipold RW, Conradi R, Martin E, Motsch J. Efficacy of ultrasound imaging in obstetric epidural anesthesia. J Clin Anesth 2002; 14: 169-175.

11. Grau T, Leipold RW, Horter J, Martin E, Motsch J. Colour Doppler imaging of the interspinous and epidural space. Eur J Anaesthesiol 2001; 18: 706-712.

12. Grau T, Leipold RW, Horter J, Conradi R, Martin E, Motsch $\mathrm{J}$. The lumbar epidural space in pregnancy: visualization by ultrasonography. Br J Anaesth 2001; 86: 798-804.

13. Grau T, Leipold RW, Conradi R, Martin E, Motsch J. The visualisation of dura perforation and blood patches with ultrasound. Anasthesiol Intensivmed Notfallmed Schmerzther 2002; 37: 149-153.

14. Grau T, Leipold RW, Conradi R, Martin E. Ultrasound control for presumed difficult epidural puncture. Acta Anaesthesiol Scand 2001; 45: 766-771.

15. Grau T, Leipold R, Conradi R, Martin E, Motsch J. Ultrasonography and peridural anesthesia. Technical possibilities and limitations of ultrasonic examination of the epidural space. Anaesthesist 2001; 50: 94-101.

16. Karmakar MK, Li X, Ho AM, Kwok WH, Chui PT. Realtime ultrasound-guided paramedian epidural access: evaluation of a novel in-plane technique. Br J Anaesth 2009; 102: 845-854.

17. Conroy PH, Luyet C, McCartney CJ, McHardy PG. Realtime ultrasound-guided spinal anaesthesia: a prospective observational study of a new approach. Anesthesiol Res Pract 2013; 2013: 525818.

18. Tran D, Kamani AA, Al-Attas E, Lessoway VA, Massey S, Rohling RN. Single-operator real-time ultrasound-guidance to aim and insert a lumbar epidural needle. Can J Anaesth 2010; 57: 313-321.

19. Brinkmann S, Tang R, Sawka A, Vaghadia H. Single-operator real-time ultrasound-guided spinal injection using SonixGPS: a case series. Can J Anaesth 2013; 60: 896-901.

20. Niazi AU, Chin KJ, Jin R, Chan VW. Real-time ultrasoundguided spinal anesthesia using the SonixGPS ultrasound guidance system: a feasibility study. Acta Anaesthesiol Scand 2014; 58: 875-881.

21. Wong SW, Niazi AU, Chin KJ, Chan VW. Real-time ultrasound-guided spinal anesthesia using the SonixGPS(R) needle tracking system: a case report. Can J Anaesth 2013; 60: 50-53.

22. Ansari T, Yousef A, El Gamassy A, Fayez M. Ultrasoundguided spinal anaesthesia in obstetrics: is there an advantage over the landmark technique in patients with easily palpable spines? Int J Obstet Anesth 2014; 23: 213-216.

23. Chin KJ, Karmakar MK, Peng P. Ultrasonography of the adult thoracic and lumbar spine for central neuraxial blockade. Anesthesiology 2011; 114: 1459-1485.

24. Perlas A. Evidence for the use of ultrasound in neuraxial blocks. Reg Anesth Pain Med 2010; 35(2 Suppl): S43-S46.

25. Halpern SH, Banerjee A, Stocche R, Glanc P. The use of ultrasound for lumbar spinous process identification: A pilot study. Can J Anaesth 2010; 57: 817-822.

26. Schlotterbeck H, Schaeffer R, Dow WA, Touret Y, Bailey $\mathrm{S}$, Diemunsch P. Ultrasonographic control of the puncture 
level for lumbar neuraxial block in obstetric anaesthesia. $\mathrm{Br}$ J Anaesth 2008; 100: 230-234.

27. Brinkmann S, Tang R, Vaghadia H, Sawka A. Assessment of a real-time ultrasound-guided spinal technique using SonixGPS in human cadavers. Can J Anaesth 2012; 59: 11561157.

28. Margarido CB, Arzola C, Balki M, Carvalho JC. Anesthesiologists' learning curves for ultrasound assessment of the lumbar spine. Can J Anaesth 2010; 57: 120-126.

29. Chin KJ, Ramlogan R, Arzola C, Singh M, Chan V. The utility of ultrasound imaging in predicting ease of performance of spinal anesthesia in an orthopedic patient population. Reg Anesth Pain Med 2013; 38: 34-38.

30. Chin KJ, Perlas A, Chan V, Brown-Shreves D, Koshkin A, Vaishnav V. Ultrasound imaging facilitates spinal anesthesia in adults with difficult surface anatomic landmarks. Anesthesiology 2011; 115: 94-101.

31. Arzola C, Davies S, Rofaeel A, Carvalho JC. Ultrasound using the transverse approach to the lumbar spine provides reliable landmarks for labor epidurals. Anesth Analg 2007; 104: 1188-1192.

32. Balki M, Lee Y, Halpern S, Carvalho JC. Ultrasound imaging of the lumbar spine in the transverse plane: the correlation between estimated and actual depth to the epidural space in obese parturients. Anesth Analg 2009; 108: 1876-1881.

33. Vallejo MC, Phelps AL, Singh S, Orebaugh SL, Sah N. Ultrasound decreases the failed labor epidural rate in resident trainees. Int J Obstet Anesth 2010; 19: 373-378.

34. Broadbent CR, Maxwell WB, Ferrie R, Wilson DJ, GawneCain M, Russell R. Ability of anaesthetists to identify a marked lumbar interspace. Anaesthesia 2000; 55: 1122-1126.
35. Watson MJ, Evans S, Thorp JM. Could ultrasonography be used by an anaesthetist to identify a specified lumbar interspace before spinal anaesthesia? Br J Anaesth 2003; 90: 509-511.

36. Furness G, Reilly MP, Kuchi S. An evaluation of ultrasound imaging for identification of lumbar intervertebral level. Anaesthesia 2002; 57: 277-280.

37. Bron JL, van Royen BJ, Wuisman PI. The clinical significance of lumbosacral transitional anomalies. Acta Orthop Belg 2007; 73: 687-695.

38. Chin KJ, Perlas A, Singh M, et al. An ultrasound-assisted approach facilitates spinal anesthesia for total joint arthroplasty. Can J Anaesth 2009; 56: 643-650.

39. Weed JT, Taenzer AH, Finkel KJ, Sites BD. Evaluation of pre-procedure ultrasound examination as a screening tool for difficult spinal anaesthesia*. Anaesthesia 2011; 66: 925930.

40. Hampl K, Steinfeldt T, Wulf H. Spinal anesthesia revisited: toxicity of new and old drugs and compounds. Curr Opin Anaesthesiol 2014; 27: 549-555.

41. Belavy D, Sunn N, Lau Q, Robertson T. Absence of neurotoxicity with perineural injection of ultrasound gels: assessment using an animal model. BMC Anesthesiol 2013; 13: 18.

42. Lee PJ, Tang R, Sawka A, Krebs C, Vaghadia H. Brief report: real-time ultrasound-guided spinal anesthesia using Taylor's approach. Anesth Analg 2011; 112: 1236-1238.

43. Tiouririne M, Nguyen S, Hossack JA, Owen K, William Mauldin F Jr. Handheld real-time volumetric imaging of the spine: technology development. J Med Eng Technol 2014; 38: 100-103. 